\title{
Asociación entre las características antropométricas y la condición física en jugadores de baloncesto en silla de ruedas
}

\author{
Relationship between the anthropometric characteristics \\ and physical fitness in wheelchair basketball players
}

\author{
Ander Romarate, Cristina Granados, Aitor Iturricastillo, Miren Lizundia y Javier Yanci Irigoyen*
}

Facultad de Educación y Deporte, Universidad del País Vasco, UPVIEHU, Vitoria-Gasteiz (España).

\begin{abstract}
Resumen: Los objetivos de este estudio fueron, en primer lugar, describir las características antropométricas y de la condición física en jugadores de baloncesto en silla de ruedas (BSR), en segundo lugar, analizar la asociación entre las características antropométricas y la condición física y, en tercer lugar, estudiar la asociación existente entre las distintas capacidades físicas. Once jugadores $(33,82 \pm 10,17$ años, 74,50 \pm 5,18 cm talla sentados $)$ de la Primera División de la Liga española de BSR participaron en este estudio. Las características antropométricas (masa corporal, pliegues cutáneos, perímetros y diámetros corporales), y la condición física (capacidad de aceleración, cambio de dirección, fuerza muscular y capacidad aeróbica) fueron medidas. Los resultados mostraron una asociación significativa entre el sumatorio de pliegues y el tiempo en el test Pick-up ( $r=-0,64 \pm 0,35 \mathrm{CL}, \mathrm{p}<$ $0,05)$, el test maximal pass (Mpass) $(\mathrm{r}=0,74 ; \pm 0,28 \mathrm{CL}, \mathrm{p}<0,01)$ y el test de lanzamiento de balón medicinal (Bmed) $(0,73 ; \pm 0,29 \mathrm{CL}, \mathrm{p}<0,05)$. Los resultados obtenidos en el presente estudio muestran que las características antropométricas podrían estar relacionadas con la capacidad de cambio de dirección y la fuerza muscular en jugadores de BSR de categoría nacional (primera división española).

Palabras clave: Test de campo, composición corporal, pliegues cutáneos, rendimiento, discapacidad.
\end{abstract}

Abstract: The three main objectives of this paper were, in first place, to describe the anthropometric characteristics and the physical conditions and in wheelchair basketball (WB) players, in second place, to analyze the relation between the anthropometric characteristics and the physical conditions, and in third place, to study the association between the different physical conditions. Eleven players $(33,82 \pm 10,17$ years, $74,50 \pm 5,18 \mathrm{~cm}$ sitting size) from the First Division of the Spanish WB League took part in this study. The anthropometric characteristics (body mass, skin folds and body perimeters and diameters), and physical condition (acceleration, change of direction, muscular strength and aerobic capacity) were measured. The obtained results shown an significant association between the summation of the folds and the time in the Pick-up test $(\mathrm{r}=-0,64 \pm 0,35 \mathrm{CL}, \mathrm{p}<0,05)$, in the maximal pass test (Mpass) $(\mathrm{r}=0,74 ; \pm 0,28 \mathrm{CL}, \mathrm{p}<0,01)$ and the test of the medicine ball throw $(0,73 ; \pm 0,29 \mathrm{CL}, \mathrm{p}<0,05)$. The results obtained in the present study show that the anthropometric characteristics could be related to the change direction ability and muscle strength in WB players of national category (Spanish First division).

Key words: Field test, body composition, skin folds, efficiency, disability.

\section{Introducción}

El baloncesto en silla de ruedas (BSR) es un deporte paralímpico desde los Juegos de Roma en 1960 (Weissland, Faupin, Borel, \& Leprêtre, 2015). Esta modalidad deportiva está regulada a nivel internacional por la Federación Internacional de Baloncesto en Silla de Ruedas (International Wheelchair Basketball Federation, IWBF) y a nivel nacional por la Federación Española de Deportes de Personas con Discapacidad Física (FEDDF) (Yanci, Iturricastillo, \& Granados, 2014). Es un deporte practicado en cientos de países y la cantidad de participantes en todo el mundo asciende a los 30.000 deportistas (Cavedon, Zancanaro, \& Milanese, 2015). El BSR es una modalidad muy popular entre personas con discapacidad física (Santos, Krishnan, Alonso, \& Greve, 2016) y está diseñada principalmente para jugadores cuyas características físicas no les permitan correr, saltar y/o pivotar, como puede ser el caso de personas con lesión medular, amputaciones o

Dirección para correspondencia [Correspondence address]: Javier Yanci Irigoyen. E-mail: javier.yanci@ehu.es diferentes afectaciones del sistema musculo esquelético (Goosey-Tolfrey \& Leicht, 2013).

La importancia de las características antropométricas en el rendimiento deportivo ha sido analizada en multitud de ocasiones en diferentes modalidades deportivas (Gorostiaga et al., 2009; Granados et al., 2015). Sin embargo, existen pocos estudios que analicen las características antropométricas de jugadores de BSR (Cavedon et al., 2015; Chapman, Fulton, \& Gough, 2010). Estudios previos han analizado las características antropométricas de estos jugadores, midiendo la talla sentado, la masa corporal, los pliegues cutáneos, el perímetro del brazo relajado y el perímetro del brazo contraído (GooseyTolfrey, Batterham, \& Tolfrey, 2003; Granados et al., 2015; Yanci et al., 2015). Así mismo, Cavedon et al. (2015) analizaron también otras características antropométricas como la talla sentado, la talla con los brazos extendidos, los pliegues cutáneos y el porcentaje de grasa corporal en jugadores jóvenes de BSR. Teniendo en cuenta que existen pocos estudios al respecto y la relevancia que pueden tener las características 
antropométricas en este grupo de población podría ser interesante continuar investigando sobre las características antropométricas de jugadores de BSR.

Desde un punto de vista de las demandas del juego, el BSR está caracterizado por la multitud de acciones intermitentes que se dan durante los partidos, donde se intercalan acciones de alta y baja intensidad (Iturricastillo, Granados, \& Yanci, 2015). Además, durante el juego, los deportistas deben de realizar acciones específicas como la propulsión o el manejo de la silla, aceleraciones, deceleraciones y cambios de dirección con y sin balón (Wang, Chen, Limroongreungrat, \& Change, 2005). Por tanto, debido a la alta exigencia física y fisiológica de esta modalidad, la condición física de jugadores de BSR también ha sido analizada anteriormente en varias investigaciones, mediante diferentes test de laboratorio (Goosey-Tolfrey \& Leicht, 2013; Weissland et al., 2015) y test de campo (De Groot, Balvers, Kouwenhoven, \& Janssen, 2012; Molik et al., 2013; Vanlandewijck et al., 2004; Yanci et al., 2015). Concretamente, mediante test de campo se han analizado la capacidad de aceleración, la capacidad de cambiar de dirección, la fuerza muscular y la capacidad cardiovascular de jugadores de BSR (Cavedon et al., 2015; Yanci et al., 2015).

A pesar de que en otros deportes de equipo se ha analizado ampliamente la relación existente entre las características antropométricas y la condición física de los jugadores (Silvestre, West, Maresh, \& Kraemer, 2006; Wong, Chamari, Dellal, \& Wisloff, 2009) o la relación existente entre los distintos test de rendimiento (Gorostiaga et al., 2009; Mann, Ivey, Mayhew, Schumacher, \& Brechue, 2016), pocos estudios han analizado si las capacidades físicas están relacionadas con las características antropométricas (Cavedon et al., 2015; Chapman et al., 2010) o la relación existente entre los distintos test de condición física (Cavedon et al., 2015; De Groot et al., 2012; Molik et al., 2013; Yanci et al., 2015) en jugadores de BSR. De esta manera, estudiar estas asociaciones podría aportar información relevante a entrenadores, preparadores físicos, nutricionistas o distintos profesionales relacionados con el BSR, con el fin de poder conocer si es relevante realizar programas nutricionales y de control de las características antropométricas en los jugadores para mejorar su rendimiento físico, así como que capacidades físicas podrían ser las que mayor relación tienen entre ellas.

Por lo tanto, los objetivos de este estudio fueron, en primer lugar, describir las características antropométricas y la condición física de jugadores de BSR, en segundo lugar, analizar la asociación entre las características antropométricas y la condición física y, en tercer lugar, estudiar la relación existente entre las distintas capacidades físicas.

\section{Método}

\section{Participantes}

Once jugadores de BSR (edad = 33,82 \pm 10,17 años, talla sentado $=74,50 \pm 5,18 \mathrm{~cm}$ ), que competían en un equipo de Primera División de la Liga española de BSR, participaron en este estudio. Todos los jugadores tenían al menos 7 años de experiencia en BSR. Del total de los participantes, cinco jugadores estaban clasificados en la clase funcional 1, un jugador en la clase 2, un jugador en la clase 3,5, dos jugadores en la clase 4 y dos jugadores en la clase 4,5 , todos ellos clasificados siguiendo el protocolo del Comité de Clasificación de la International Wheelchair Basketball Federation (IWBF). Antes de comenzar con el estudio, a todos los jugadores se les informó sobre los riesgos y beneficios de la participación en el mismo, firmaron el consentimiento informado y podían retirarse del estudio en cualquier momento. El estudio fue aprobado por el Comité de Ética para la Investigación con Seres Humanos (CEISH, código 201200037882) de la Universidad del País Vasco (UPV/EHU).

\section{Procedimiento}

La batería de test se realizó en dos días al final de la pretemporada, una semana antes del inicio de la temporada competitiva oficial. Todas las sesiones de test se realizaron en la cancha de baloncesto donde entrenaba el equipo, en el horario habitual de entrenamiento, entre las 19 y las $21 \mathrm{~h}$. El primer día, los jugadores realizaron los test de aceleración (test de sprint en 5 y $20 \mathrm{~m}$, con y sin balón) y los test de agilidad (T-test y Pick-up test). El segundo día (48 horas después) se realizaron las mediciones de composición corporal, los test de fuerza (maximal pass, lanzamiento de balón medicinal y handgrip) y el test de resistencia (Yo-Yo Intermittent Recovery Level 1 de $10 \mathrm{~m}$ ). Se recomendó a los participantes que los días previos a las pruebas no realizaran actividad física intensa. Los jugadores fueron instruidos para realizar las pruebas a la máxima intensidad posible. Antes de las sesiones de test, todos los participantes realizaron un calentamiento que consistía en 5 min de desplazamiento a baja intensidad con la silla de ruedas, 4 aceleraciones de $10 \mathrm{~m}$ y 4 aceleraciones de $20 \mathrm{~m}$ con cambio de dirección. Todos los jugadores realizaron las pruebas con su silla de competición habitual.

\section{Mediciones}

Composición corporal: Se midió en cada jugador la masa corporal $(\mathrm{kg})$, los pliegues cutáneos $(\mathrm{mm})$, los perímetros $(\mathrm{cm})$ y los diámetros $(\mathrm{cm})$ corporales. La masa corporal fue obtenida mediante una báscula electrónica (Seca ${ }^{\circledR}$ Instruments Ltd., Hamburg, Germany) y los cuatro pliegues cutá- 
neos (tríceps, subescapular, suprailíaco y abdominal) fueron medidos con un piclómetro (Harpender Lange ${ }^{\ominus}$, Cambridge, MA, USA) siguiendo las indicaciones especificadas por Goosey et al. (2003) para jugadores de BSR. Se calculó también el sumatorio de los cuatro pliegues ( $\sum$ Pliegues) (Granados et al., 2015).

Test de sprint en 5 y 20 m (con y sin balón): Los jugadores realizaron 3 aceleraciones máximas de $20 \mathrm{~m}$ sin balón y otras 3 de 20 m con balón (De Groot et al., 2012), con 2 min de descanso entre cada repetición. Los participantes se situaban inicialmente en una posición de $0,5 \mathrm{~m}$ con respecto a la primera fotocélula y comenzaban cuando ellos lo consideraban oportuno. El temporizador se activaba automáticamente cuando el deportista sobrepasaba la primera fotocélula y se registró el tiempo a los 5 y 20 m tanto en las aceleración sin balón (S5m y S20m) como con balón (SB5m y SB20m) (Iturricastillo et al., 2015). En los esprintes realizados con balón, los participantes debían cumplir el reglamento marcado por la IWBF. El tiempo (s) empleado en cada sprint se registró utilizando tres fotocélulas (Microgate Polifemo Radio Ligth ${ }^{\oplus}$, Bolzano, Italia) colocadas al inicio $(0 \mathrm{~m})$, intermedio $(5 \mathrm{~m})$ y al final $(20 \mathrm{~m})$, a una altura de $0,4 \mathrm{~m}$. El mejor tiempo fue utilizado en el análisis estadístico.

Test de cambio de dirección, T-test: Los participantes completaron un circuito en forma de $\mathrm{T}$ siguiendo el protocolo utilizado anteriormente con jugadores de BSR por Yanci et al. (2015). Los participantes realizaron la prueba 3 veces con un descanso entre repeticiones de $3 \mathrm{~min}$. La distancia total recorrida fue de $36,56 \mathrm{~m}$ y la altura de los 4 conos era de 0,3 m. Para el registro del tiempo (s), se situó una fotocélula (Microgate Polifemo Radio Ligth ${ }^{\oplus}$, Bolzano, Italia) en la línea de salida y llegada. El mejor registro fue utilizado en el análisis estadístico.

Test de cambio de dirección, Pick-up: Siguiendo el protocolo descrito por De Groot et al. (2012), desde una posición de parado, cada jugador debía comenzar a impulsar la silla y recoger 4 balones situados en el suelo de la cancha, dos veces con la mano izquierda y dos veces con la mano derecha. Después de recoger el balón, debían situarlo en el regazo y cada participante tenía que propulsar la silla por lo menos dos veces antes de soltar el balón. Para registrar el tiempo total (s) empleado en la ejecución del test, se utilizaron dos fotocélulas (Microgate Polifemo Radio Ligth ${ }^{\oplus}$, Bolzano, Italia) situadas al inicio y al final de la prueba. Todos los participantes realizaron la prueba tres veces con un descanso de 3 min entre repeticiones. El mejor tiempo fue utilizado en el análisis estadístico.

Test Handgrip (Hand): Se midió la fuerza isométrica del antebrazo en la mano dominante (Iturricastillo et al., 2015) mediante un dinamómetro hidráulico de mano portátil (5030J1, Jamar ${ }^{\oplus}$, Sammons Preston, Nottinghamshire, Reino Unido). La prueba se realizó en posición de sentado en la silla de ruedas y con el brazo extendido. El protocolo de test consistió en realizar 3 contracciones isométricas máximas de $5 \mathrm{~s}$, con un descanso de $60 \mathrm{~s}$ entre cada repetición (Iturricastillo et al., 2015). El mejor registro de los tres fue utilizado en el análisis estadístico.

Maximal pass (Mpass): Los participantes se situaron en una línea marcada, con las ruedas delanteras de la silla encima de la línea y realizaron cinco lanzamientos bilaterales de balón lo más lejos posible (De Groot et al., 2012). Se registró la distancia $(\mathrm{m})$ entre la línea marcada y el lugar donde el balón tocó el suelo por primera vez. La distancia media obtenida en los cinco lanzamientos fue utilizado en el análisis estadístico (De Groot et al., 2012).

Test de lanzamiento de balón medicinal (BMed): Situados en la misma posición que en el Mpass, los participantes lanzaron un balón de $5 \mathrm{~kg}$ lo más lejos posible (De Groot et al., 2012). Cada participante realizó tres lanzamientos y se registró la distancia $(\mathrm{m})$ entre la línea marcada y el lugar donde el balón entró en contacto con el suelo por primera vez. Para el análisis estadístico se utilizó el mejor registro de los tres.

Test de resistencia Yo-Yo Intermittent Recovery Level 1 10 m (YYIR1 10m): Se utilizó la versión 1 del test YYIR1 $10 \mathrm{~m}$ previamente descrito por Yanci et al. (2015) para jugadores de BSR. La velocidad fue marcada mediante un sistema de audio previamente programado. Se consideraba el test por finalizado cuando un participante no llegaba a la línea marcada en el tiempo estipulado por segunda vez (evaluación objetiva) o cuando el participante consideraba que no podía continuar con la prueba (evaluación subjetiva). La distancia total recorrida, la frecuencia cardíaca máxima (FCmax) (Polar Team Sport System ${ }^{\oplus}$, Polar Electro Oy, Finland) y la concentración de lactato en sangre (LAC) al final de la prueba (Lactate Pro LT-1710 ${ }^{\oplus}$, ArkRay Inc Ltd, Kyoto, Japón) fueron registrados. Al finalizar el test YYIR1 10 m, se registró el esfuerzo percibido respiratorio (RPEres) y muscular (RPEmus) (Granados et al., 2015) de cada participante.

\section{Análisis estadístico}

Los resultados se presentan como media \pm desviación típica (DT) de la media, aportando también los datos mínimos y máximos. Para determinar la relación entre las características antropométricas y la condición física se utilizó la correlación de Pearson (r). Para la interpretación de las magnitudes de las correlaciones se utilizó la siguiente escala: menor que 0,1 , trivial; de 0,1 a 0,3 , baja; de 0,3 a 0,5 , moderada; de 0,5 hasta 0,7 , alta; 0,7-0,9, muy alta; mayor que 0,9, casi perfecta (Hopkins, Marshall, Batterham, \& Hanin, 2009). Además se calculó el límite de confianza ( \pm CL) al $90 \%$ y las probabilidades de ser ciertas las asociaciones (Hopkins et al., 2009). El análisis estadístico se realizó con el programa Statistical 
Package for Social Sciences (SPSS Inc, versión 23,0 Chicago; IL, EE.UU). El nivel de significación estadística fue establecido en $\mathrm{p}<0,05$.

\section{Resultados}

En la tabla 1 se muestran los resultados correspondientes a las características antropométricas (pliegues cutáneos, perímetros del brazo y diámetros de codo y de muñeca) de todos los jugadores de BSR participantes en este estudio.

Tabla 1. Características antropométricas de los jugadores de baloncesto en silla de ruedas (BSR).

\begin{tabular}{lcccc}
\hline & Mínimo & Máximo & Media & DT \\
\hline Pliegues (mm) & & & & \\
$\quad$ Tríceps & 5,0 & 21,0 & 13,2 & 4,4 \\
$\quad$ Subescapular & 9,2 & 40,0 & 21,5 & 10,2 \\
$\quad$ Suprailíaco & 7,4 & 31,8 & 17,17 & 7,7 \\
$\quad$ Abdominal & 11,3 & 51,0 & 33,1 & 11,4 \\
$\quad$ Ppliegues & 32,9 & 132,0 & 84,8 & 27,1 \\
Perímetros (cm) & & & & \\
$\quad$ Brazo relajado & 31,0 & 38,2 & 34,1 & 2,4 \\
$\quad$ Brazo contraído & 32,2 & 40,8 & 36,1 & 2,4 \\
Diámetros (cm) & & & & \\
Codo & 7,0 & 8,6 & 7,6 & 0,5 \\
$\quad$ Muñeca & 5,4 & 6,1 & 5,8 & 0,2 \\
\hline
\end{tabular}

DT = desviación típica, $\sum$ pliegues = sumatorio de cuatro pliegues.

En la tabla 2 se exponen los resultados de los test de rendimiento físico (sprint, sprint con balón, CODA, fuerza y capacidad cardiovascular) obtenidos por los jugadores de BSR participantes en este estudio.
Tabla 2. Resultados de los jugadores de baloncesto en silla de ruedas (BSR) en los test de sprint, sprint con balón, CODA, fuerza y capacidad cardiovascular.

\begin{tabular}{lcccc}
\hline & Mínimo & Máximo & Media & DT \\
\hline Sprint & & & & \\
S5m (s) & 1,6 & 2,0 & 1,8 & 0,2 \\
S20m (s) & 4,9 & 6,0 & 5,4 & 0,4 \\
Sprint con balón & & & & \\
SB5m (s) & 1,6 & 2,5 & 1,9 & 0,2 \\
SB20m (s) & 5,2 & 7,0 & 5,9 & 0,5 \\
CODA & & & & \\
T-test (s) & 13,4 & 17,4 & 15,3 & 1,2 \\
Pick-up (s) & 11,0 & 17,4 & 12,6 & 1,3 \\
Fuerza & & & & \\
Hand (kg) & 41,4 & 80,0 & 55,9 & 11,5 \\
MPass (m) & 6,8 & 13,7 & 10,6 & 2,5 \\
BMed (m) & 2,9 & 5,6 & 4,3 & 0,9 \\
YYIR1 10m & & & & \\
LAC (mmol/l) & 4,9 & 13,7 & 9,6 & 2,9 \\
FCmax (l/min) & 145,0 & 204,0 & 182,7 & 15,4 \\
Distancia (m) & 620,0 & 1800,0 & 1225,5 & 419,0 \\
RPEres & 3,0 & 10,0 & 6,2 & 2,1 \\
RPEmus & 4,0 & 9,0 & 6,9 & 1,9 \\
\hline
\end{tabular}

DT = desviación típica, S5m = sprint en 5 metros, S20m = sprint en 20 metros, SB5m = sprint en 5 metros con balón, SB20m = sprint en 20 metros con balón, CODA= capacidad de cambio de dirección, Hand = handgrip, MPass = maximal pass, $\mathrm{BMed}=$ balón medicinal, YYIR $110 \mathrm{~m}=$ test Yo-Yo Intermitent Recovery nivel 1 de 10 $\mathrm{m}, \mathrm{LAC}=$ concentración de lactato, FCmax $=$ frecuencia cardiaca máxima, RPEres = esfuerzo percibido respiratorio, $\mathrm{RPEmus}=\mathrm{es}-$ fuerzo percibido muscular.

La asociación entre las características antropométricas y la condición física de los jugadores de BSR se muestra en la tabla 3. Se encontró una asociación negativa entre el pliegue del tríceps y el rendimiento en el Pick-up $(\mathrm{p}<0.05)$. De la misma forma, se obtuvo una asociación significativa entre el pliegue subescapular y el rendimiento en el test MPass $(\mathrm{p}<0.05)$ o en el BMed ( $\mathrm{p}<0.05)$. También se encontró una asociación significativa entre el pliegue suprailíaco y el rendimiento en el BMed $(\mathrm{p}<0.05)$. Por otra parte, se observó una asociación negativa entre el sumatorio de pliegues y el Pick-up, el MPass $y$ el BMed $(\mathrm{p}<0.05)$. Los resultados obtenidos en este estudio también mostraron una asociación entre el brazo relajado y contraído con el RPEmus declarado por los jugadores después de realizar el test YYIR1 $10 \mathrm{~m}(\mathrm{p}<0.05)$. Finalmente, los resultados mostraron una relación significativa entre el diámetro del codo con la FCmáx y el RPEres $(\mathrm{p}<0.05)$. 
Tabla 3. Asociación entre las características antropométricas y la condición física de los jugadores de baloncesto en silla de ruedas (BSR).

\begin{tabular}{|c|c|c|c|c|c|c|c|c|c|c|c|c|c|c|}
\hline & $\mathrm{S} 5 \mathrm{~m}$ & $\mathrm{~S} 20 \mathrm{~m}$ & $\mathrm{SB} 5 \mathrm{~m}$ & SB20m & T-test & t Pick-up & Hand & MPass & BMed & LAC & FCmax & Dist & RPEres & RPEmus \\
\hline \multicolumn{15}{|l|}{ Pliegues } \\
\hline Tríceps & NS & NS & NS & NS & NS & $\begin{array}{c}-0,72 ; \pm 0,28^{*} \\
0 / 1 / 99 \#\end{array}$ & NS & NS & NS & NS & NS & NS & NS & NS \\
\hline Subescapular & NS & NS & NS & NS & NS & NS & NS & $\begin{array}{c}0,66 ; \pm 0,34^{*} \\
97 / 2 / 1 \#\end{array}$ & $\begin{array}{c}0,63 ; \pm 0,35^{*} \\
96 / 3 / 1 \#\end{array}$ & NS & NS & NS & NS & NS \\
\hline Suprailíaco & NS & NS & NS & NS & NS & NS & NS & NS & $\begin{array}{c}0,60 ; \pm 0,37^{*} \\
95 / 4 / 1 \#\end{array}$ & NS & NS & NS & NS & NS \\
\hline Abdominal & NS & NS & NS & NS & NS & NS & NS & $\begin{array}{c}0,63 ; \pm 0,35^{*} \\
96 / 3 / 1 \#\end{array}$ & NS & NS & NS & NS & NS & NS \\
\hline$\sum$ pliegues & NS & NS & NS & NS & NS & $\begin{array}{c}-0,64 ; \pm 0,35^{*} \\
1 / 2 / 97 \#\end{array}$ & NS & $\begin{array}{c}0,74 ; \pm 0,28^{* *} \\
99 / 1 / 0 \#\end{array}$ & $\begin{array}{c}0,73 ; \pm 0,29^{*} \\
99 / 1 / 0 \#\end{array}$ & NS & NS & NS & NS & NS \\
\hline \multicolumn{15}{|l|}{ Perímetros } \\
\hline Brazo relajado & NS & NS & NS & NS & NS & NS & NS & NS & NS & NS & NS & NS & NS & $\begin{array}{c}-0,61 ; \pm 0,37 \\
1 / 3 / 96 \#\end{array}$ \\
\hline Brazo contraído & NS & NS & NS & NS & NS & NS & NS & NS & NS & NS & NS & NS & NS & $\begin{array}{c}-0,62 ; \pm 0,36 \\
1 / 3 / 96 \#\end{array}$ \\
\hline \multicolumn{15}{|l|}{ Diámetros } \\
\hline Codo & NS & NS & NS & NS & NS & NS & NS & NS & NS & NS & $\begin{array}{c}-0,65 ; \pm 0,34^{*} \\
1 / 2 / 97 \#\end{array}$ & NS & $\begin{array}{c}-0,64 ; \pm 0,35^{*} \\
1 / 2 / 97 \#\end{array}$ & NS \\
\hline Muñeca & NS & NS & NS & NS & NS & NS & NS & NS & NS & NS & NS & NS & NS & NS \\
\hline
\end{tabular}

S5m = sprint en 5 metros, S20m = sprint en 20 metros, SB5m = sprint en 5 metros con balón, SB20m= sprint en 20 metros con balón, Hand $=$ Handgrip, MPass = maximal pass, BMed = balón medicinal, LAC = lactato, FCmáx $=$ Frecuencia cardiaca máxima, Dist $=$ Dis tancia, RPEres = esfuerzo percibido respiratorio, RPEmus = esfuerzo percibido muscular, $\sum$ pliegues $=$ Sumatorio de pliegues, NS $=$ no significativa. Correlación significativa $*(\mathrm{p}<0.05),{ }^{* *}(\mathrm{p}<0.01)$. \# Muy probable.

Atendiendo a las asociaciones entre los distintos test de condición física, se observó una alta asociación entre la capacidad de aceleración en 5 y $20 \mathrm{~m}$ sin balón y 5 y $20 \mathrm{~m}$ con balón $(\mathrm{r}=0,76 ; \pm 0,26$, muy probable / 0,92; $\pm 0,10$, extremadamente probable, $\mathrm{p}<0,01)$. También se observó una alta asociación entre la capacidad de aceleración con y sin balón y el rendimiento en el T-test (Figura 1). Sin embargo, no se obtuvo asociación significativa $(\mathrm{p}>0,05)$ entre la capacidad de aceleración con y sin balón (5 y $20 \mathrm{~m}$ ) y el Pick-up. Por otro lado, tanto el T-test como el Pick-up correlacionaron signifi- cativamente con los test de fuerza $(\mathrm{r}=-0,61 ; \pm 0,37$, muy probable / - 0,79; $\pm 0,24$, muy probable, $\mathrm{p}<0,05)$ y los distintos test de fuerza correlacionaron de forma significativa entre sí $(r=0,66 ; \pm 0,34$, muy probable / 0,93; $\pm 0,09$, extremadamente probable, $\mathrm{p}<0,01)$. Finalmente, se observaron asociaciones significativas entre la capacidad de aceleración (S5, S20, SB5 y SB20) o el T-test con la distancia recorrida en el test YYIR1 $10 \mathrm{~m}(\mathrm{r}=-0,62 ; \pm 0,36$, muy probable / $-0.86 ; \pm 0,17$, extremadamente probable, $\mathrm{p}<0.05$ ) (Figura 2). 

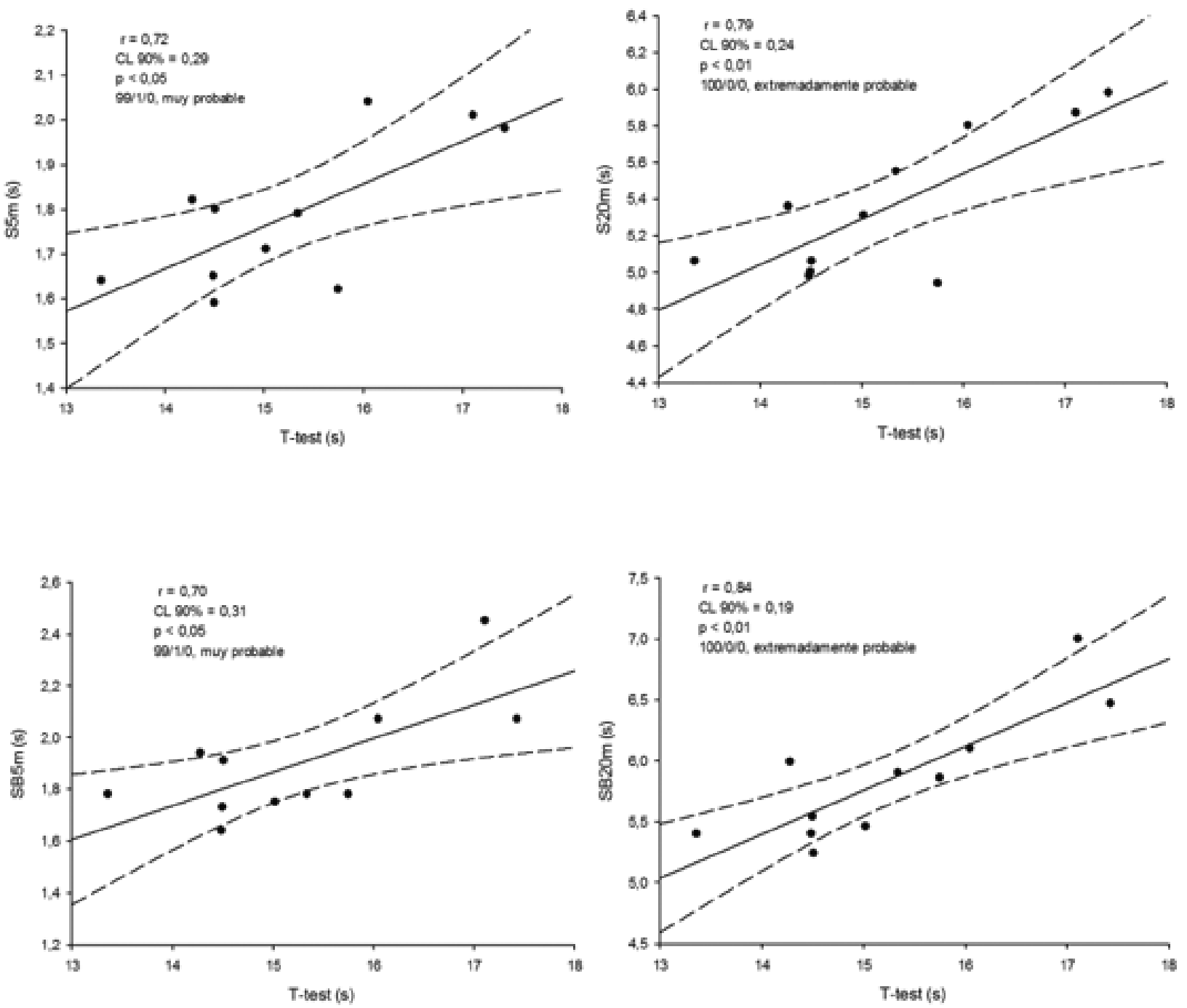

Figura 1. Asociaciones entre los resultados en el sprint de 5 metros y el T-test (1A), el sprint de 20 metros y el T-test (1B), el sprint de 5 metros con balón y el T-test (IC) y el sprint de 20 metros con balón y el T-test (1D) obtenidas por los jugadores de baloncesto en silla de ruedas (BSR).

S5m = sprint de 5 metros, S20m = sprint de 20 metros, SB5m = sprint de 5 metros con balón, SB20m = sprint de 20 metros con balón, CL $90 \%$ = límite de confianza al 90\%. 

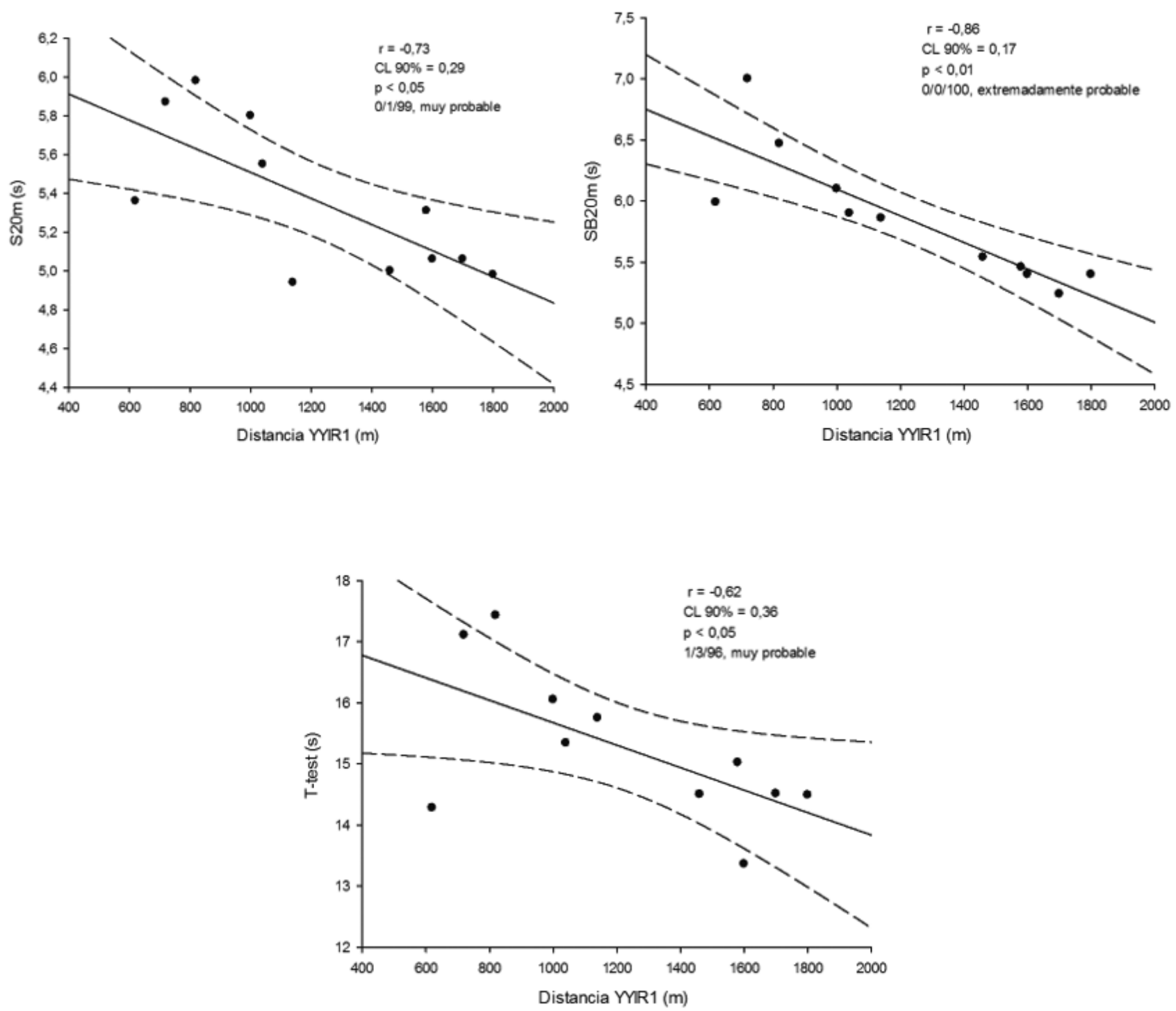

Figura 2. Asociaciones entre los resultados en el sprint de 20 metros y la distancia recorrida en el YYIR1 10m (2A), en el sprint de 20 metros con el balón y la distancia recorrida en el YYIR1 10m (2B) y en el T-test y la distancia recorrida en el YYIR1 10m (2C) obtenidas por los jugadores de baloncesto en silla de ruedas (BSR).

S20m = sprint de 20 metros, YYIR1 = test Yo-Yo Intermitent Recovery nivel 1, SB20m = sprint de 20 metros con balón, CL 90\% = límite de confianza al $90 \%$.

\section{Discusión}

Los objetivos de este estudio fueron, en primer lugar, describir las características antropométricas y la condición física en jugadores de BSR, en segundo lugar, analizar la asociación entre las características antropométricas y la condición física, y en tercer lugar, estudiar la asociación existente entre las distintas capacidades físicas. Conocer la relación entre las características antropométricas y la condición física de jugadores de BSR puede ser interesante para comprobar si la composición corporal puede afectar al rendimiento físico de los jugadores. Anteriormente, pocos estudios han analizado la asociación entre las características antropométricas y la condición física en jugadores de BSR (Cavedon et al., 2015; Chapman et al., 2010). Los resultados obtenidos en nuestro estudio mostraron que los jugadores con mayor sumatorio de pliegues, obtenían un mejor resultado en el test de Pick-up, MPass y BMed. Asimismo, se observaron asociaciones significativas entre el perímetro de brazo contraído y relajado con el RPEmus después de realizar el YYIR1 10m. Atendiendo a las asociaciones entre los distintos test de condición física, se obtuvo una asociación significativa entre el T-test o Pick-up 
con el test de handgrip y entre los test de sprint o el T-test con el YYIR1 10m. De la misma manera, los resultados mostraron una alta asociación entre los test de sprint y el T-test.

Las características antropométricas son parte de un complejo de cualidades que afectan al rendimiento deportivo (Aragoné \& Casajús, 1991) y es por ello que deben ser tenidas en cuenta. En este estudio los valores antropométricos obtenidos son inferiores a los obtenidos en otros estudios realizados con jugadores de BSR de un nivel competitivo superior o similar (Chapman et al., 2010; Granados et al., 2015; Iturricastillo et al., 2015). Algunas investigaciones anteriores han observado que los jugadores de mayor nivel competitivo (Granados et al., 2015; Yanci et al., 2015) o de mayor edad (Cavedon et al., 2015) muestran valores más altos de sumatorio de pliegues. Por lo tanto, parece ser que las características antropométricas pueden estar afectadas tanto por el nivel competitivo como por la edad de los jugadores de BSR. Por este motivo, podría ser interesante analizar si es necesario controlar la composición corporal de los jugadores a lo largo de la temporada, mediante pautas nutricionales y programas específicos de entrenamiento. Concretamente Iturricastillo et al. (2015), observaron que el sumatorio de pliegues en jugadores de BSR de alto nivel se reducía durante una temporada competitiva $(69,08 \pm 24,97 \mathrm{~mm}$ vs. $64,73 \pm 33,59$ $\mathrm{mm}$, principio y final de temporada, respectivamente). En futuros estudios podría ser interesante analizar la evolución de las características antropométricas en jugadores de BSR tras la aplicación de programas específicos de entrenamiento y control nutricional así como su influencia en el rendimiento deportivo.

Por otro lado, se ha observado que el análisis del perfil físico de los deportistas es un factor determinante en el rendimiento deportivo (Gabbett, 2016). Los resultados obtenidos en este estudio en la mayor parte de las capacidades analizadas son similares a los resultados obtenidos por Yanci et al. (2015) en jugadores de la misma categoría competitiva (Primera División española). Sin embargo, son mejores que los resultados obtenidos en jugadores de BSR de la Tercera División española (J. Yanci et al., 2015), en jugadores juveniles de BSR de la liga italiana (Cavedon et al., 2015) y en jugadores de distintas categorias holandesas de BSR (De Groot et al., 2012). En la misma línea, en un estudio realizado por Granados et al. (2015), también se observó que los jugadores de mayor categoría competitiva tenían un mejor rendimiento en distintos test de condición física. Este aspecto nos lleva a pensar que una adecuada condición física de los jugadores de BSR puede ser importante para alcanzar elevados niveles de rendimiento deportivo. Sin embargo, estos resultados hay que tomarlos con cautela, ya que la utilización de distintos protocolos, sistemas de medición o momentos de la temporada de los estudios analizados pueden haber condicionado los resultados obtenidos.
La asociación entre las características antropométricas y la condición física en jugadores de baloncesto ha sido analizada en anteriores estudios (Delextrat \& Cohen, 2009; Köklü, Alemdaroğlu, Koçak, Erol, \& Fındıkoğlu, 2011). Sin embargo, esta asociación ha sido menos estudiada en BSR (Cavedon et al., 2015; Chapman et al., 2010). Los resultados de nuestro estudio mostraron que los jugadores con un mayor sumatorio de pliegues tenían un mejor rendimiento en el Pick-up, MPass y BMed. Estos resultados son contrarios a los obtenidos tanto en baloncesto convencional (Köklü et al., 2011), como en baloncesto en silla de ruedas (Cavedon et al., 2015). Concretamente en BSR, con jugadores de BSR del Campeonato juvenil Italiano, Cavedon et al. (2015) no obtuvieron ninguna asociación significativa entre las características antropométricas (longitud y perímetro de las extremidades, pliegues cutáneos o la estatura sentado) y distintos test de rendimiento físico ( $\mathrm{S} 5 \mathrm{~m}, \mathrm{~S} 20 \mathrm{~m}$, test de capacidad cardiovascular, pass for accuracy, spot-shot, lay-ups y Mpass). En la misma línea, Chapman et al. (2010) concluyeron que las características antropométricas (talla sentado, masa corporal, perímetro del brazo y sumatorio de pliegues), no estaban asociados de forma significativa con el rendimiento físico (capacidad de aceleración, agilidad y capacidad aeróbica) en jugadores de elite de BSR. Nuestro estudio es el primer trabajo en BSR en el que se han obtenido asociaciones entre las características antropométricas y la condición física. Por lo tanto, debido a que los resultados de los estudios analizados sobre la asociación entre las características antropométricas y la condición física en jugadores de BSR son contradictorios y escasos, son necesarios más estudios al respecto que analicen si existe una asociación entre la composición corporal y el rendimiento físico en esta modalidad deportiva.

La asociación entre los distintos test de condición física ha sido muy analizada en deportes convencionales pero no tanto en modalidades de deporte adaptado y consecuentemente en el BSR (Molik et al., 2013). Los resultados del presente estudio mostraron una alta asociación entre la capacidad de aceleración con o sin balón y el T-test, pero no con el Pick-up, tal y como también se había observado en estudios anteriores (De Groot et al., 2012). Posiblemente, la asociación entre la capacidad de aceleración y el T-test y su ausencia con el Pickup, ambos test de capacidad de cambio de dirección, puede ser debido a que en el caso del Pick-up, las acciones técnicas a realizar (controlar la silla con el balón, recoger el balón del suelo, propulsar la silla y deshacerse del balón), cobren una gran importancia, por lo que la capacidad de aceleración puede jugar un papel menos relevante en este test. No obstante, los resultados obtenidos en este estudio mostraron una buena asociación entre el T-test o Pick-up con la fuerza muscular. Estos resultados concuerdan con los obtenidos por Granados et al. (2015) en jugadores de BSR. En la misma línea, Molik et al. (2013) observaron una asociación entre los 
test de lanzamiento y el slalom en jugadores de la selección canadiense de BSR. Por lo tanto, se sugiere que ambos test de cambio de dirección pueden tener una importante influencia de la fuerza muscular. Teniendo esto en cuenta, puede ser muy interesante para los entrenadores y los técnicos realizar un entrenamiento de fuerza específico con el fin de mejorar la CODA de los jugadores de BSR, ya que esta capacidad es una de las más relevantes para el rendimiento en competición (De Groot et al., 2012).

La capacidad cardiovascular también es crucial para obtener un adecuado rendimiento en los partidos de BSR (Molik et al., 2013). Los resultados del presente estudio también mostraron una buena asociación entre la capacidad de aceleración o el T-test con la distancia recorrida en el YYIR1 10m. Un estudio previo también observó una alta correlación entre un test de sprint $20 \mathrm{~m}$ o un test de slalom con un test de resistencia (De Groot et al., 2012). Las altas asociaciones encontradas en la mayoría de estudios entre los test de aceleración o CODA y los test de resistencia pueden poner de manifiesto que estas dos capacidades pueden afectar al rendimiento en los test de resistencia en jugadores de BSR. Concretamente, el test utilizado en el presente estudio, el YYIR1 10m, fue un test adaptado del deporte convencional (Krustrup et al., 2003). Las asociaciones significativas encontradas entre la capacidad de aceleración o el T-test con el test YYIR1 10m nos pueden llevar a pensar que posiblemente el rendimiento en el YYIR1 10m esté influenciado en gran medida por la capacidad de aceleración y el manejo de la silla. Por tanto, sería interesante analizar si el YYIR1 10m es un test adecuado para medir realmente la capacidad cardiovascular en los jugadores de BSR, o si está excesivamente influenciado por otros aspectos del rendimiento como son la capacidad de aceleración, la fuerza, la capacidad anaeróbica o la técnica de propulsión de la silla y la capacidad de cambiar de dirección.

\section{Conclusiones}

Aunque en estudios anteriores con jugadores de BSR no se habían encontrado asociaciones significativas entre las carac- terísticas antropométricas y la condición física, los resultados obtenidos en este estudio nos muestran que las características antropométricas están relacionadas con la capacidad de cambiar de dirección y la fuerza muscular en jugadores de BSR de nivel nacional (primera división española).

Teniendo en cuenta las características antropométricas pueden estar asociadas al rendimiento en la capacidad de cambiar de dirección y en la fuerza muscular, y que estas dos capacidades son relevantes para el rendimiento en BSR, uno de los objetivos del entrenamiento en BSR debería de ir encaminado a mejorar las características antropométricas de los jugadores.

Debido a que el número de estudios publicados en la literatura científica que han analizado esta asociación es muy reducido, podría ser interesante profundizar en este aspecto para poder determinar si la aplicación de distintos protocolos nutricionales y métodos de entrenamiento pueden influir en las características antropométricas y consecuentemente en las capacidades físicas.

\section{Agradecimientos}

Queremos agradecer al C.D. Zuzenak por ofrecernos la posibilidad de realizar este estudio de investigación y en concreto a los entrenadores y a los jugadores participantes en el mismo. Los autores agradecen el apoyo del subproyecto Enfoque de método mixto en el análisis de rendimiento (en entrenamiento y competición) en el deporte de élite y academia [PGC2018-098742-B-C33] (2019-2021) [del Ministerio de Ciencia, Innovación y Universidades (MCIU), la Agencia Estatal de Investigación (AEI) y el Fondo Europeo de Desarrollo Regional (FEDER)], que forma parte del proyecto coordinado New approach of research in physical activity and sport from mixed methods perspective (NARPAS_MM) [SPGC201800X098742CV0].

\section{Referencias}

1. Aragonés, M., \& Casajús, J. (1991). Modificaciones antropométricas debidas al entrenamiento. Estudios longitudinales. Archivos de Medicina Del Deporte, 8(32), 345-353.

2. Cavedon, V., Zancanaro, C., \& Milanese, C. (2015). Physique and performance of young wheelchair basketball players in relation with classification. PLOS ONE, 10(11), 1-20.

3. Chapman, D., Fulton, S., \& Gough, C. (2010). Anthropometric and physical performance characteristics of elite male wheelchair basketball athletes. Journal of Strength and Conditioning Research, 24, 1.

4. De Groot, S., Balvers, I. J. M., Kouwenhoven, S. M., \& Janssen, T. W. J. (2012). Validity and reliability of tests determining performancerelated components of wheelchair basketball. Journal of Sports Sciences,
30(9), 879-87.

5. Delextrat, A., \& Cohen, D. (2009). Strength, power, speed, and agility of women basketball players according to playing position. Journal of Strength and Conditioning Research, 23(7), 1974-1981.

6. Gabbett, T. J. (2016). Influence of fatigue on tackling ability in rugby league players: Role of muscular strength, endurance, and aerobic qualities. PLOS ONE, 11(10), 1-11.

7. Goosey-Tolfrey, V. L., Batterham, A. M., \& Tolfrey, K. (2003). Scaling behavior of VO2peak in trained wheelchair athletes. Medicine and Science in Sports and Exercise, 35(12), 2106-2111.

8. Goosey-Tolfrey, V. L., \& Leicht, C. A. (2013). Field-based physiological testing of wheelchair athletes. Sports Medicine, 43(2), 77-91. 
9. Gorostiaga, E. M., Llodio, I., Ibañez, J., Granados, C., Navarro, I., Ruesta, M., \& Izquierdo, M. (2009). Differences in physical fitness among indoor and outdoor elite male soccer players. European Journal of Applied Physiology, 106(4), 483-491.

10. Granados, C., Yanci, J., Badiola, A., Iturricastillo, A., Otero, M., Olasagasti, J., \& Gil, S. M. (2015). Anthropometry and performance in wheelchair basketball. Journal of Strength and Conditioning Research, 29(7), 1812-1820.

11. Hopkins, W. G., Marshall, S. W., Batterham, A. M., \& Hanin, J. (2009). Progressive statistics for studies in sports medicine and exercise science. Medicine and Science in Sports and Exercise, 41(1), 3-12.

12. Iturricastillo, A., Granados, C., \& Yanci, J. (2015). Changes in body composition and physical erformance in wheelchair basketball players during a competitive season. Journal of Human Kinetics, 48(1), 157-165.

13. Köklü, Y., Alemdaroğlu, U., Koçak, F. Ü., Erol, a E., \& Fındıkoğlu, G. (2011). Comparison of chosen physical fitness characteristics of Turkish professional basketball players by division and playing position. Journal of Human Kinetics, 30(12), 99-106.

14. Krustrup, P., Mohr, M., Amstrup, T., Rysgaard, T., Johansen, J., Steensberg, A., \& Bangsbo, J. (2003). The Yo-Yo Intermittent Recovery test : physiological response, reliability, and validity. Medicine and Science in Sports and Exercise, 35(4), 697-705.

15. Mann, J. B., Ivey, P. A., Mayhew, J. L., Schumacher, R. M., \& Brechue, W. F. (2016). Relationship between agility tests and hort sprints. Journal of Strength and Conditioning Research, 30(4), 893-900.

16. Molik, B., Laskin, J. J., Kosmol, A., Marszałek, J., Morgulec-Adamowicz, N., \& Frick, T. (2013). Relationships between anaerobic performance, field tests, and functional level of elite female wheelchair basketball athletes. Human Movement, 14(4), 366-371.

17. Santos, S. D. S., Krishnan, C., Alonso, A. C., \& Greve, J. M. D. (2016). Trunk function correlates positively with wheelchair basketball player classification. American Journal of Physical Medicine and Rehabilitation, $0(6), 1-10$
18. Silvestre, R., West, C., Maresh, C. M., \& Kraemer, W. J. (2006). Body composition and physical performance in men's soccer: a study of a National Collegiate Athletic Association Division I team. Journal of Strength and Conditioning Research, 20(1), 177-183.

19. Vanlandewijck, Y. C., Evaggelinou, C., Daly, D. J., Verellen, J., Van Houtte, S., Aspeslagh, V., \& Zwakhoven, B. (2004). The relationship between functional potential and field performance in elite female wheelchair basketball players. Journal of Sports Sciences, 22(7), 668-675.

20. Wang, Y. T., Chen, S., Limroongreungrat, W., \& Change, L. S. (2005) Contributions of selected fundamental factors to wheelchair basketball performance. Medicine and Science in Sports and Exercise, 37(1), 130137.

21. Weissland, T., Faupin, A., Borel, B., \& Leprêtre, P. M. (2015). Comparison between 30-15 intermittent fitness test and multistage field test on physiological responses in wheelchair basketball players. Frontiers in Physiology, 6(12), 1-8.

22. Wong, P.-L., Chamari, K., Dellal, A., \& Wisloff, U. (2009). Relationship between anthropometric and physiological characteristics in youth soccer players. Journal of Strength and Conditioning Research, 23(18), 1204-1210.

23. Yanci, J., Granados, C., Otero, M., Badiola, A., Olasagasti, J., Bidaurrazaga-Letona, I., \& Gil, S. M. (2015). Sprint, agility, strength and endurance capacity in wheelchair basketball players. Biology of Sport, 32(1), 71-78

24. Yanci, J., Iturricastillo, A., \& Granados, C. (2014). Heart rate and body temperature response of wheelchair basketball players in small-sided games. International Journal of Performance Analysis in Sport, 14(2), 535-544.

25. Yanci, J., Iturricastillo, A., \& Granados, C. (2015). Analisis de la condición física de jugadores nacionales de baloncesto en silla atendiendo a la clasificación funcional. RICYDE: Revista Internacional de Ciencias del Deporte, 11(41), 226-244. 